\title{
"Influence of cross-shelf water transport on nutrients and phytoplankton in the East China Sea: a model study" published in Ocean Sci., 7, 27-43, 2010
}

\section{Zhao ${ }^{1}$ and X. Guo ${ }^{2}$}

${ }^{1}$ Physical Oceanography Laboratory, Ocean University of China, 238 Songling Road, Qingdao 266100, China

${ }^{2}$ Center for Marine Environmental Studies, Ehime University, 2-5 Bunkyo-Cho, Matsuyama 790-8577, Japan

In the paper "Influence of cross-shelf water transport on nutrients and phytoplankton in the East China Sea: a model study" by L. Zhao and X. Guo (Ocean Sci., 7, 27-43, 2011) an error occurred in the affiliation of X. Guo.

$\mathrm{X}$. Guo is only affiliated to the second affiliation, Center for Marine Environmental Studies, Ehime University, 2-5 Bunkyo-Cho, Matsuyama 790-8577, Japan, as seen above. 